\title{
Genetic variability of VEGF pathway genes in six randomized phase III trials assessing the addition of bevacizumab to standard therapy
}

\author{
Sanne de Haas $\cdot$ Paul Delmar - Aruna T. Bansal - Matthieu Moisse • \\ David W. Miles • Natasha Leighl - Bernard Escudier • Eric Van Cutsem • \\ Peter Carmeliet $\cdot$ Stefan J. Scherer · Celine Pallaud • Diether Lambrechts
}

Received: 13 February 2014 / Accepted: 5 July 2014/Published online: 11 July 2014

(C) Springer Science+Business Media Dordrecht 2014

\begin{abstract}
Background Despite extensive translational research, no validated biomarkers predictive of bevacizumab treatment outcome have been identified.

Methods We performed a meta-analysis of individual patient data from six randomized phase III trials in colorectal, pancreatic, lung, renal, breast, and gastric cancer to explore the potential relationships between 195 common genetic variants in the vascular endothelial growth factor (VEGF) pathway and bevacizumab treatment outcome.

Results The analysis included 1,402 patients (716 bevacizumab-treated and 686 placebo-treated). Twenty variants were associated $(P<0.05)$ with progression-free
\end{abstract}

Sanne de Haas and Paul Delmar have contributed equally to this work.

Electronic supplementary material The online version of this article (doi:10.1007/s10456-014-9438-1) contains supplementary material, which is available to authorized users.

S. de Haas · P. Delmar · C. Pallaud

F. Hoffmann-La Roche, Basel, Switzerland

\section{A. T. Bansal}

Acclarogen Ltd, St John's Innovation Centre, Cambridge, UK

M. Moisse · P. Carmeliet · D. Lambrechts $(\bowtie)$

Vesalius Research Center, VIB, Herestraat 49 Box 912,

3000 Louvain, Belgium

e-mail: Diether.Lambrechts@vib-kuleuven.be

M. Moisse - D. Lambrechts

Laboratory of Translational Genetics, Department of Oncology,

University of Leuven, Herestraat 49 Box 912, 3000 Louvain,

Belgium

D. W. Miles

Mount Vernon Cancer Centre, Northwood, UK survival (PFS) in bevacizumab-treated patients. Of these, 4 variants in EPAS1 survived correction for multiple testing $(q<0.05)$. Genotype-by-treatment interaction tests revealed that, across these 20 variants, 3 variants in VEGF$C$ (rs12510099), EPAS1 (rs4953344), and IL8RA (rs2234671) were potentially predictive $(P<0.05)$, but not resistant to multiple testing $(q>0.05)$. A weak genotypeby-treatment interaction effect was also observed for rs699946 in VEGF-A, whereas Bayesian genewise analysis revealed that genetic variability in $V H L$ was associated with PFS in the bevacizumab arm $(q<0.05)$. Variants in $V E G F-A, E P A S 1$, and $V H L$ were located in expression quantitative loci derived from lymphoblastoid cell lines, indicating that they affect the expression levels of their respective gene.

Conclusions This large genetic analysis suggests that variants in VEGF-A, EPAS1, IL8RA, VHL, and VEGF$C$ have potential value in predicting bevacizumab treatment outcome across tumor types. Although these associations

\author{
N. Leighl \\ Department of Medicine, Princess Margaret Hospital, Toronto, \\ ON, Canada \\ B. Escudier \\ Institut Gustave Roussy, Villejuif, France \\ E. Van Cutsem \\ Digestive Oncology, University Hospitals Leuven and KU \\ Leuven, Louvain, Belgium \\ S. J. Scherer \\ Genentech Inc., South San Francisco, CA, USA
}


did not survive correction for multiple testing in a genotype-by-interaction analysis, they are among the strongest predictive effects reported to date for genetic variants and bevacizumab efficacy.

Keywords Anti-angiogenesis - Bevacizumab - Treatment outcome - Genetic variant Predictive and prognostic biomarker

\section{Introduction}

Bevacizumab, a humanized monoclonal antibody that binds to vascular endothelial growth factor (VEGF), was the first anti-VEGF-specific drug to be approved in the clinic. The addition of bevacizumab to standard therapy has been shown to significantly improve outcome in patients with metastatic colorectal [1], non-small cell lung [2], breast [3-5], renal [6], and ovarian [7-9] cancer, and recurrent glioblastoma [10]. Although anti-angiogenic drugs have changed clinical practice in several cancers, various clinical challenges remain [11]. For instance, after initial response, adaptive escape mechanisms ultimately lead to disease progression in most patients $[12,13]$. There is remarkable heterogeneity in the timing of angiogenic escape, with some patients relapsing almost immediately and others having prolonged periods of stabilized disease [14]. A current challenge, therefore, is to identify and validate markers predictive of bevacizumab treatment outcome. Despite concerted efforts using a broad spectrum of biological sample types [15-17], a validated marker predictive of treatment outcome for bevacizumab therapy has not been identified so far.

As host factors may influence angiogenesis, it is plausible that genetic variability may underlie differences in response to bevacizumab. The characterization of common genetic variability therefore represents a logical focus in bevacizumab biomarker research. Various potential predictive genetic markers have been identified in clinical trials evaluating bevacizumab in several tumor types [1826] but these analyses, generally using single-trial datasets, are limited by the number of available samples, heterogeneity in the selection of single-nucleotide polymorphisms (SNPs), and lack of understanding of the functional consequences of these genetic markers.

The aim of this study was to identify genetic variants associated with bevacizumab treatment outcome in patients with advanced cancer regardless of tumor type. Although it is not yet clear to what extent markers predictive of treatment response are shared between cancer types, a clear advantage of such an approach is that patients participating in various phase III clinical trials can be combined and much larger patient numbers can be assessed. Here, we pooled patients from six clinical studies in breast, colorectal, pancreatic, gastric, lung, and renal cancers, thereby performing the largest genetic study to date aiming to predict bevacizumab efficacy.

\section{Patients and methods}

Patient population

The analysis included samples from patients treated in six randomized phase III trials of bevacizumab in colorectal cancer (NO16966), pancreatic cancer (BO17706; AViTA), advanced or recurrent non-squamous non-small cell lung cancer (BO17704; AVAiL), metastatic renal cell cancer (BO17705; AVOREN), HER2-negative metastatic breast cancer (BO17708; AVADO), and advanced gastric cancer (BO20904; AVAGAST). The designs and primary clinical results (efficacy and safety) for these trials have been published previously [1, 4, 6, 27-29]. Four of the trials met their primary objectives of improving overall survival (OS) and/or progression-free survival (PFS). However, although AViTA and AVAGAST demonstrated improved PFS (secondary end point), the primary end point of OS in these trials did not improve with the addition of bevacizumab to standard chemotherapy. As expected, owing to the fact that different tumor types were assessed, median values and censoring rates for PFS and OS varied considerably between the trials (Table 1).

The genetic analyses were performed on a subset of patients who consented to participate in a genetic substudy, donated a blood sample from which DNA could be successfully extracted and genotyped, and self-reported "white" ethnicity (with the aim of limiting false positives by using an ethnically homogeneous patient population).

\section{Laboratory methods}

Peripheral blood samples were collected in K2EDTA Vacutainer tubes (BD, Franklin Lakes, NJ, USA). Germ line DNA was extracted from the precipitated leukocyte cell fraction. Genotyping was performed at the Vesalius Research Center, Leuven, Belgium, with MassARRAY iPLEX Gold (Sequenom, San Diego, CA, USA), as reported previously [23].

We selected SNPs in the following 15 genes involved in the VEGF-A pathway: VEGF-A, the VEGF-A homologs [placental growth factor $(P l G F), V E G F-B, V E G F-C$, and $V E G F-D$ (also known as c-fos-induced growth factor or $F I G F)$, VEGF receptor-1 (VEGFR-1 or FLT1), VEGF receptor-2 (VEGFR-2 or KDR) and $V E G F$ receptor-3 (VEGFR-3 or FLT4), regulators of hypoxia [hypoxia inducible factor-1 $\alpha$ (HIFlA), HIF-2 $\alpha$ (EPAS1), factor inhibiting HIF-1A (FIHI), von Hippel-Lindau tumor 


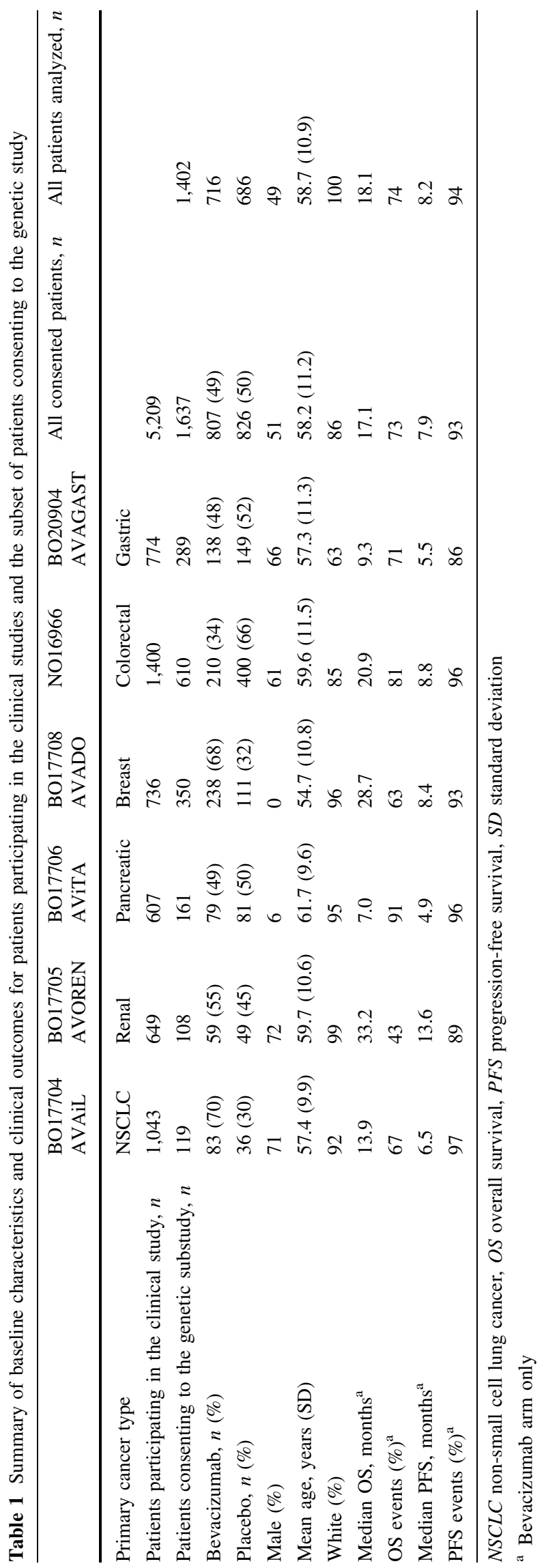

suppressor $(V H L)$ ], and the oxygen sensors [prolyl hydroxylase domain-containing protein 1 (EGLN2), 2 (EGLN1), and 3 (EGLN3)]. A detailed description of how SNPs were selected in these genes has been published previously [23]. Briefly, genomic sequences $5 \mathrm{~kb}$ upstream of the translation start site and downstream of the $3^{\prime}$ polyadenylation site of each gene were used to select SNPs from the HapMap database (Phase 2 Public release number 22). Common SNPs with a minor allele frequency $\geq 0.1$ and pairwise correlation coefficients $\left(r^{2}\right) \leq 0.8$ were selected using the SNP Tagger approach of the Haploview software package $[30,31]$. Overall, we selected 211 tagging SNPs in these VEGF pathway genes, as well as 10 genetic variants previously associated with bevacizumab efficacy. Fifteen SNPs known to increase patients' susceptibility to hypertension and thrombosis were also included. These were not analyzed for their effect on bevacizumab treatment outcome, but for correlation with bevacizumab-induced hypertension; results were published recently [32]. After testing for minor allele frequency and the homogeneity of the observed allele frequency (Supplementary Fig. 1), 195 SNPs were included in the analysis (Supplementary Table 1).

Circulating VEGF-A and VEGF-C concentrations were measured in plasma collected from 119 healthy individuals with self-reported Flemish ethnicity for three generations using Human Quantikine ELISA kits (R\&D Systems) according to the manufacturer's instructions. Expression and genotype data of 856 lymphoblastoid cell lines derived from healthy female twins of the MuTHER study [33] were used to assess SNP-gene associations in expression quantitative loci (eQTLs) using the Genevar [34] platform.

\section{Statistical methods}

Individual patient data were pooled for the meta-analysis. Potential correlations between markers and clinical outcome were assessed in the subset of bevacizumab-treated patients, the subset of placebo-treated patients, and in all patients irrespective of treatment. The end point of primary interest was PFS; OS was a secondary end point. Four covariates were pre-specified for these analyses: geographic region, study, bevacizumab dose, and chemotherapy backbone. Backwards stepwise regression identified additional covariates: Eastern Cooperative Oncology Group (ECOG) performance status (0 vs. 1/2), lactate dehydrogenase level (normal vs. abnormal), baseline serum albumin level ( $\leq 29$ vs. $>29 \mathrm{~g} / \mathrm{L}$ ), and baseline number of lesions ( $\leq 2$ vs. $>2$ ) for PFS and OS. For OS, two additional covariates were identified: alkaline phosphatase level ( $\leq 2.5$ vs. $>2.5 \times$ upper limit of normal) and gender. 
For the single-marker analyses, 195 genetic markers were first tested for single-point association with bevacizumab treatment effect using Cox proportional hazards regression within the subset of patients treated with bevacizumab. Results were then compared with tests of association in the subset of patients not treated with bevacizumab. Genotype-by-treatment interactions were used in the full set to formally test the potential predictive value of candidate markers. Prognostic characteristics were evaluated using tests of association in the full dataset. False discovery rate (FDR) $q$ values were computed to correct for multiple testing, reflecting the exploratory nature of these analyses [35].

For the genewise analysis, associations were tested on a gene-by-gene basis using an empirical Bayesian generalized linear model [36, 37]. This method was originally developed to allow the joint analysis of data from multiple functionally related genes in microarray data, and was implemented to jointly analyze multiple markers from a single gene. The method takes into account the different group sizes, i.e., the different numbers of variants tested in each gene (e.g., $>30$ variants tested in EPASI vs. 2 or 3 markers tested in most other genes). The implemented method used is available in the R-package as the globaltest. Elastic net analysis was applied to evaluate potential prognostic or predictive effects of marker combinations. A subset of 141 candidate markers with $<10 \%$ missing data was subjected to elastic net variable selection [38-40]. Elastic net analysis differs from the above analyses as it allows simultaneous analysis of clinical covariates and markers, thereby identifying the best statistical predictors for a given trait. It encompasses a penalized regression approach that carries advantages of both ridge and lasso penalties, such that it provides shrinkage and variable selection with efficient handling of highly correlated variables (multi-collinearity). Missing genotypes were imputed to the heterozygote genotype (the mode); all variables were standardized, and tuning parameters were selected by twodimensional tenfold cross-validation. In the interests of sparsity, the partial likelihood deviance was allowed to increase to its highest level such that prediction error was within one standard deviation of the minimum. To obtain a robust estimate of the multivariate model performance, all patients (irrespective of treatment) were stratified by study and randomized to a training or validation set. Elastic net analysis was performed on the training set, and the identified variables were combined into a single continuous classification signature based on the Cox proportional hazards model. The performance of the model was tested using the validation set. Patients were classified into two groups based on the median of the signature, and the two patient subgroups were compared using Kaplan-Meier estimates.

\section{Results}

Patient population

The subset of patients included in this genetic substudy comprised 1,402 patients: 716 randomized to bevacizumab and 686 randomized to placebo (Table 1). Only patients who self-reported "white" ethnicity were included in the analysis. Notably, only $60 \%$ of patients participating in the AVAGAST (BO20904) study were from Western Europe, Australia, or North America, whereas in the remaining 5 trials, $88 \%$ of patients were from Western Europe, Australia, or North America, $10 \%$ were from Eastern Europe, and $2 \%$ were from the rest of the world.

\section{Single-marker analyses}

Of the 195 variants tested, 20 were associated with PFS in bevacizumab-treated patients at an unadjusted $P<0.05$ (Table 2; variants are shown in descending order of association strength). Data for all 195 variants tested are listed in Supplementary Table 1. The strongest associations with PFS were seen for variants in EPASI and VEGF-A (12 out of $34 E P A S 1$ variants and 4 out of $15 V E G F-A$ variants had a $P<0.05$ ). The remaining associations were in $2 \mathrm{VHL}$ variants, 1 VEGF-C and 1 IL8RA variant. Notably, 4 variants in EPAS1 (rs4145836, rs11689649, rs7594278, and rs1374749), of which rs4145836 was most significantly associated with PFS [hazard ratio (HR) $0.69 ; 95 \%$ confidence interval (CI) $0.58-0.83 ; P=0.0001$ ], also survived correction for multiple testing $(q<0.05$ using FDR correction).

Genotype-by-treatment interaction tests indicated a potential predictive effect for 3 of these 20 variants: rs12510099 in $V E G F-C$ (interaction $P=0.0311$ ), rs4953344 in EPAS1 (interaction $P=0.0454$ ), and rs2234671 in IL8RA (interaction $P=0.0492$ ). None of these SNPs survived correction for multiple testing $(q>0.05)$. Kaplan-Meier curves for these individual SNPs are shown in Fig. 1. Forest plots displaying HRs in the individual studies are presented in Fig. 2. One additional variant (rs699946 in the VEGF-A promoter) showed a trend toward a potential predictive interaction effect (interaction $P=0.0907$; Table 2). The allelic HR for this SNP was 1.27 (95\% CI 1.08-1.49; $P=0.0034$ ), whereas no effect was seen in placebo-treated patients $(P=0.9110)$. The nearby rs699947 SNP in VEGF-A, which was previously identified as a predictor of bevacizumab treatment outcome in the E2100 trial in breast cancer [20], was not associated with bevacizumab treatment outcome in our analysis.

Although our primary end point of interest was PFS, similar analyses were performed for the secondary end point, OS. Eight of the 195 variants showed a potential 
Table 2 Markers with $P<0.05$ in association testing of 195 markers against PFS in white patients receiving bevacizumab, with corresponding data in placebo-treated patients. The four variants with the lowest genotype-by-treatment interaction $P$-value $(P<0.1)$ are marked in bold

\begin{tabular}{|c|c|c|c|c|c|c|c|c|c|c|c|}
\hline \multirow[t]{2}{*}{ Marker } & \multirow[t]{2}{*}{$\mathrm{Chr}$} & \multicolumn{3}{|l|}{ All white } & \multicolumn{4}{|c|}{ Bevacizumab } & \multirow{2}{*}{$\begin{array}{l}\text { Placebo } \\
P \text { value }\end{array}$} & \multirow{2}{*}{$\begin{array}{l}\text { Genotype-by- } \\
\text { treatment interaction } P\end{array}$} & \multirow[t]{2}{*}{ Gene } \\
\hline & & Position & MAF & HWE $P$ & $N$ & HR & $95 \% \mathrm{CI}$ & $P$ value & & & \\
\hline rs4145836 & 2 & 46595363 & 0.13 & 0.96 & 641 & 0.69 & $0.58-0.83$ & $1.00 \mathrm{E}-04$ & 0.0059 & 0.7874 & EPAS1 \\
\hline rs11689649 & 2 & 46617118 & 0.50 & 0.79 & 485 & 1.28 & $1.12-1.47$ & $3.00 \mathrm{E}-04$ & 0.5098 & 0.1521 & EPAS1 \\
\hline rs7594278 & 2 & 46604593 & 0.47 & 0.61 & 656 & 1.22 & $1.09-1.37$ & $6.00 \mathrm{E}-04$ & 0.1320 & 0.3731 & EPAS1 \\
\hline rs1374749 & 2 & 46596433 & 0.48 & 0.56 & 660 & 0.82 & $0.73-0.92$ & $8.00 \mathrm{E}-04$ & 0.3062 & 0.1659 & EPAS1 \\
\hline rs6753127 & 2 & 46597296 & 0.08 & 0.06 & 661 & 0.73 & $0.59-0.89$ & 0.0025 & 0.3337 & 0.2381 & EPAS1 \\
\hline rs3768730 & 2 & 46592524 & 0.47 & 0.80 & 463 & 1.25 & $1.08-1.44$ & 0.0028 & 0.2831 & 0.4392 & EPAS1 \\
\hline rs699946 & 6 & 43732669 & 0.18 & 0.30 & 542 & 1.27 & $1.08-1.49$ & 0.0034 & 0.9110 & 0.0907 & $V E G F-A$ \\
\hline rs 1678607 & 3 & 10188428 & 0.13 & 0.28 & 659 & 0.78 & $0.66-0.93$ & 0.0051 & 0.2200 & 0.4150 & $V H L$ \\
\hline rs833058 & 6 & 43731854 & 0.37 & 0.88 & 660 & 1.18 & $1.05-1.32$ & 0.0053 & 0.5404 & 0.2328 & $V E G F-A$ \\
\hline rs7565341 & 2 & 46599030 & 0.41 & 0.31 & 654 & 0.85 & $0.76-0.95$ & 0.0055 & 0.3361 & 0.2804 & EPAS1 \\
\hline rs12510099 & 4 & 177602953 & 0.09 & 0.94 & 643 & 0.73 & $0.58-0.91$ & 0.0060 & 0.9266 & 0.0311 & $V E G F-C$ \\
\hline rs3025030 & 6 & 43750587 & 0.14 & 0.12 & 636 & 1.22 & $1.04-1.43$ & 0.0124 & 0.4816 & 0.4143 & $V E G F-A$ \\
\hline rs3025039 & 6 & 43752536 & 0.15 & 0.31 & 703 & 1.21 & $1.04-1.40$ & 0.0135 & 0.8285 & 0.2277 & $V E G F-A$ \\
\hline rs2881324 & 2 & 46576894 & 0.49 & 0.27 & 327 & 0.82 & $0.69-0.96$ & 0.0153 & 0.1234 & 0.8957 & EPAS1 \\
\hline rs 1562452 & 2 & 46580444 & 0.50 & 0.59 & 661 & 0.87 & $0.77-0.97$ & 0.0155 & 0.0232 & 0.8606 & EPAS1 \\
\hline rs1642742 & 3 & 10191943 & 0.32 & 0.55 & 650 & 0.86 & $0.77-0.97$ & 0.0163 & 0.9199 & 0.1540 & $V H L$ \\
\hline rs4953344 & 2 & 46552458 & 0.16 & 0.43 & 640 & 0.83 & $0.71-0.97$ & 0.0165 & 0.4763 & 0.0454 & EPAS1 \\
\hline rs13409493 & 2 & 46588488 & 0.12 & 0.59 & 587 & 0.82 & $0.68-0.98$ & 0.0338 & 0.5773 & 0.3727 & EPAS1 \\
\hline rs2234671 & 2 & 219029108 & 0.05 & 0.28 & 695 & 1.33 & $1.02-1.73$ & 0.0376 & 0.4303 & 0.0492 & IL8RA \\
\hline rs2121267 & 2 & 46549389 & 0.45 & 0.77 & 661 & 1.13 & $1.01-1.26$ & 0.0381 & 0.8354 & 0.2354 & EPAS1 \\
\hline
\end{tabular}

$C h r$ chromosome, $C I$ confidence interval, $N$ number of patients, $M A F$ minor allele frequency, $H R$ hazard ratio, $H W E P$ Hardy-Weinberg $P$ value

association $(P<0.05)$ with $\mathrm{OS}$ in bevacizumab-treated patients (Supplementary Table 2). Three of these variants were located in VEGFR-1 (FLTI). The most distinct association (HR 1.41, $95 \%$ CI 1.15-1.72; $P=0.001$ ) was for rs 12505758, which is one of the 26 VEGFR-2 (KDR) variants tested. However, none of the 8 variants surpassed FDR correction. Genotype-by-treatment interaction tests suggested a potential predictive effect on OS for rs7987649 in VEGFR-1 (interaction $P=0.0062$ ) and rs12505758 in VEGFR-2 (interaction $P=0.0165$; Supplementary Table 2).

We also assessed the effect of these 195 variants on PFS irrespective of treatment arm. Analysis of PFS data collected from all 1,402 patients treated with either bevacizumab or placebo revealed 25 variants exhibiting $P<0.05$. Twelve of these variants were located in EPAS1 (Supplementary Table 3). Similar to the bevacizumabtreated arm, rs4145836 in EPAS1 was most significantly associated with PFS (Fig. 3a; allelic HR 0.74; $95 \%$ CI $0.65-0.84 ; P=2.2 \times 10^{-6}$ ) and survived correction for multiple testing. In 5 of 6 trials, the HRs for rs 4145836 consistently pointed in the same direction, indicating that the prognostic effect of rs4145836 was consistent across tumor types (Fig. 3b). Overall, 5 markers in EPAS1 (rs4145836, rs6712143, rs6715787, rs1562452, and rs7594278) were associated with PFS after correcting for multiple testing ( $q<0.05$ using FDR correction). Similar analyses for OS in all 1,402 patients revealed that 15 out of 195 variants were associated with OS at $P<0.05$. Six of these were in EPAS1, but none of them surpassed the multiple testing threshold. Notably, the association between rs4145836 in EPAS1 and OS (HR 0.81; $95 \%$ CI $0.71-0.94 ; P=0.0045)$ was in the same direction as observed for PFS.

Genewise analyses and elastic net analyses

Next, we complemented these single-variant analyses with a Bayesian genewise association analysis for PFS. By considering all variants located in a specific gene simultaneously, this analysis explored whether the combined effects of individual variants were associated with PFS either in the bevacizumab-treated group or in the overall patient population. Eighteen genes containing 2 or more variants were considered for this analysis. After correction for multiple testing using the FDR approach, 2 genes were associated with PFS $(q<0.05)$ in the bevacizumab arm: EPAS1 (34 variants) and VHL (2 variants). In the entire population of 1,402 patients, only EPAS1 had an FDRadjusted $q<0.05$ for PFS. In a similar genewise analysis 
Fig. 1 Kaplan-Meier curves for progression-free survival stratified by treatment arm and single-nucleotide polymorphisms. a rs 12510099 in $V E G F-C$, b rs4953344 in EPAS1, c rs2234671 in IL8RA, and d rs699946 in VEGF-A
Fig. 2 Forest plots showing the association of progression-free survival with a rs12510099 in $V E G F-C$, b rs4953344 in

EPAS1, c rs2234671 in IL8RA, and d rs699946 in VEGF-

A. $C R C$ colorectal cancer, $B C$ breast cancer, $G C$ gastric cancer, $R C C$ renal cell cancer, $P C$ pancreatic cancer, $N S C L C$ non-squamous non-small cell lung cancer
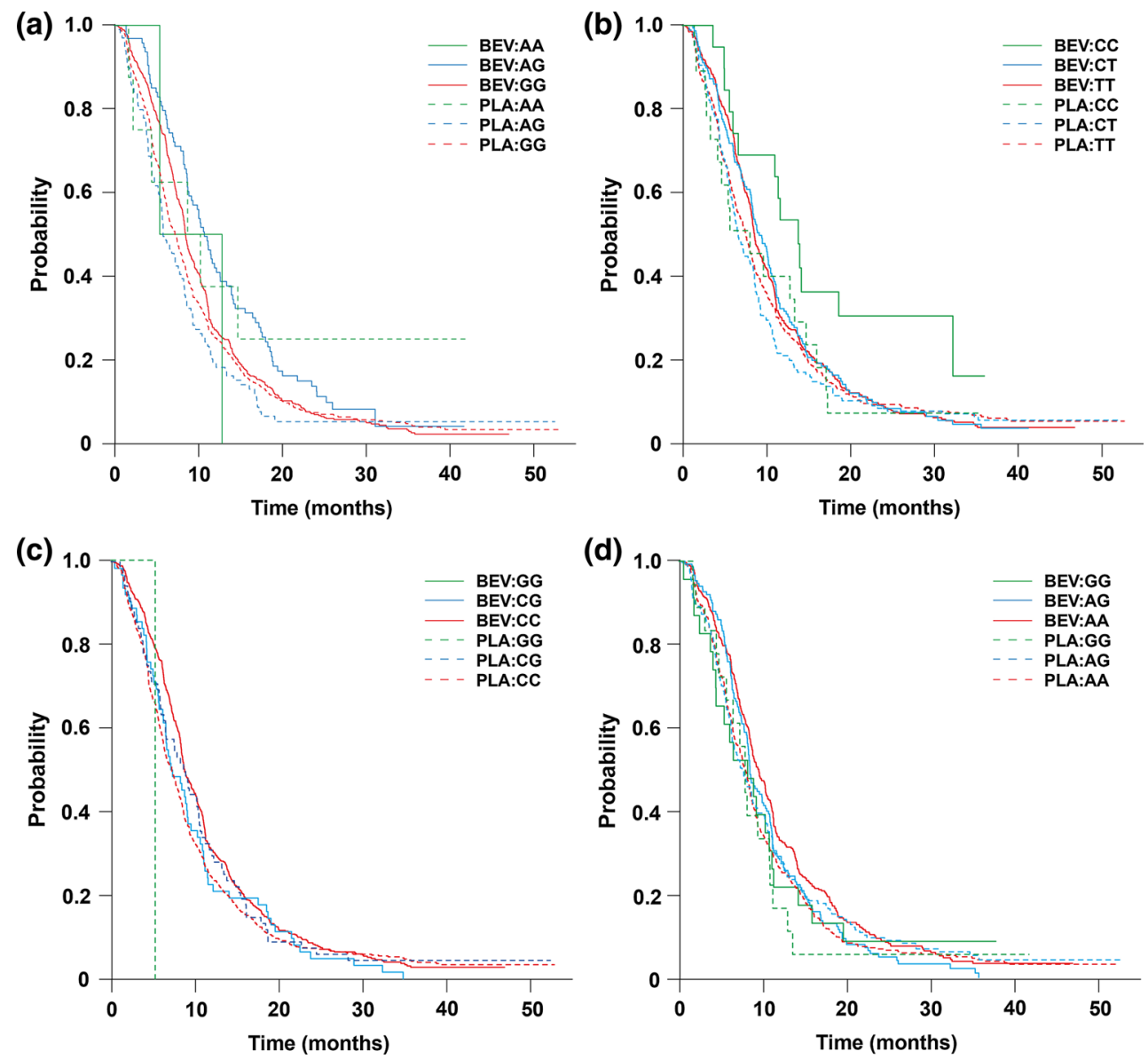

(a)

(b)

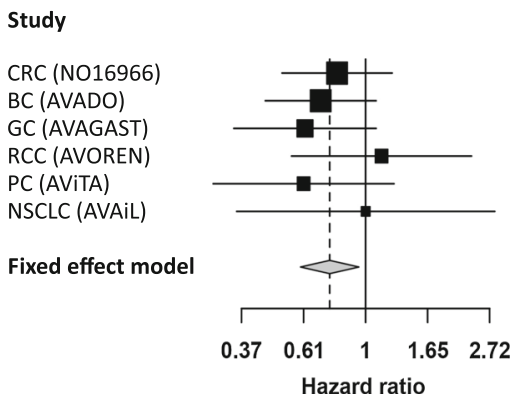

(c)

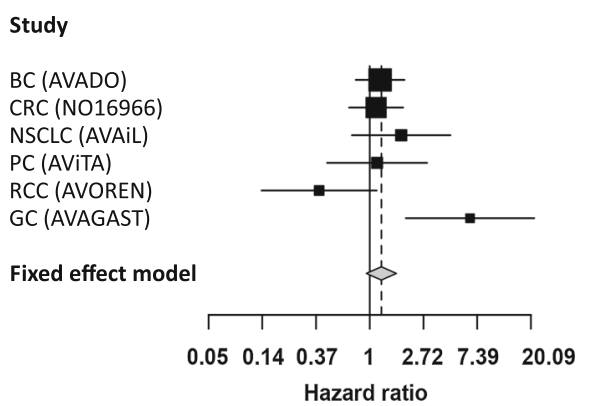

(d)

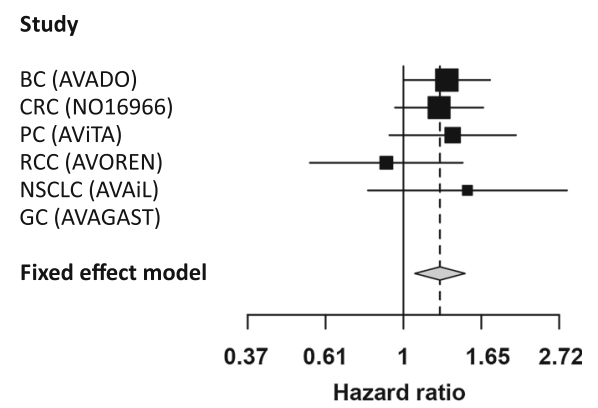




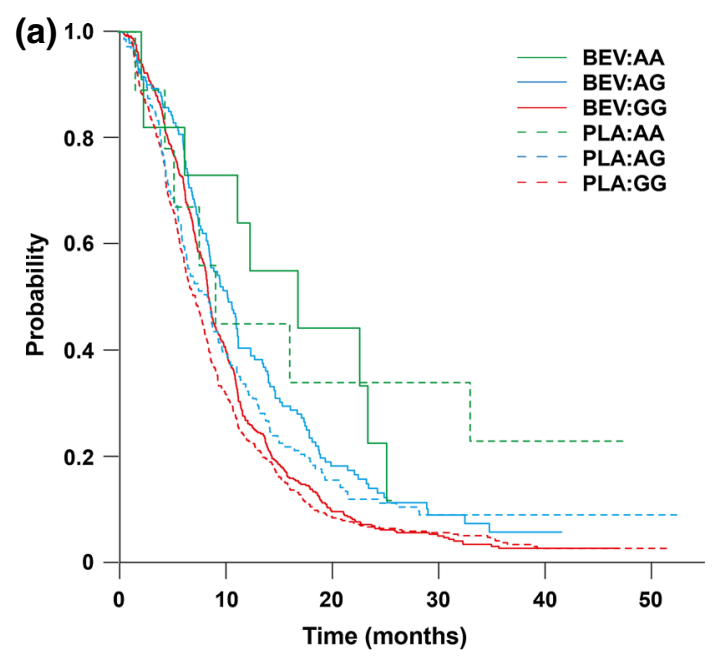

(b) Study

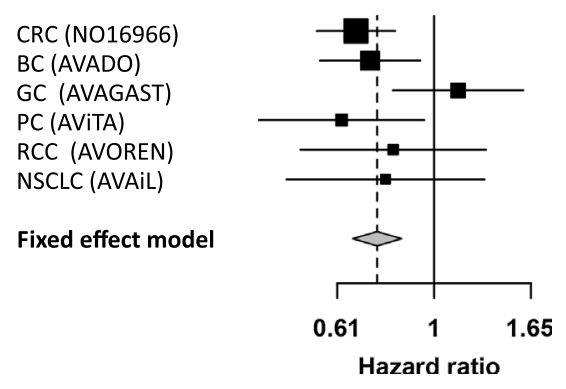

Fig. 3 a Kaplan-Meier curves for progression-free survival stratified by treatment arm and rs4145836 genotypes. b Forest plot showing the association of rs4145836 in EPAS1 with progression-free survival. $C R C$ colorectal cancer, $B C$ breast cancer, $G C$ gastric cancer, $R C C$ renal cell cancer, $P C$ pancreatic cancer, $N S C L C$ non-squamous nonsmall cell lung cancer

for OS, none of the genes had an FDR-adjusted $q<0.05$ for $\mathrm{OS}$ in either the bevacizumab-treated subset or the combination of both treatment arms.

Given the consistent prognostic effect of EPASI variants observed in the univariate and genewise analyses, a multivariate elastic net analysis was undertaken to identify a combination of genetic and clinical variables prognostic for clinical outcome. With respect to PFS, 4 clinical covariates (region, concomitant gemcitabine, ECOG performance status, and trial) and two genetic variants [rs4145836 in EPAS1 and rs3034659, the +4422 (AC)11-14 repeat in $V E G F R$-2] were identified as a signature in the training set (Supplementary Table 4a). Figure 4a shows the KaplanMeier curve in the validation set when classified by the median of this signature. The HR comparing the "above median" versus "below median" subgroups revealed a substantial and significant difference in the overall population (HR 0.57; $95 \%$ CI $0.48-0.67 ; P=2.9 \times 10^{-12}$ ) and within both individual treatment arms (bevacizumab: HR $0.59 ; 95 \%$ CI $0.47-0.73 ; P=2.2 \times 10^{-6}$; placebo: HR $0.54 ; 95 \%$ CI $\left.0.43-0.68 ; P=1.4 \times 10^{-7}\right)$. The performance of the PFS signature was also investigated with respect to OS in the validation set (Fig. 4b) and demonstrated a similar strong effect (HR 0.43; $95 \% \mathrm{CI}$ $0.36-0.52$ ).

When using the elastic net analysis for the identification of predictors for OS, 9 clinical covariates were identified in the training set as well as 8 genetic variants (rs1042886 in $P l G F$, rs 12888409 in HIF-1A, rs1870377 and rs2125489 in $V E G F R-2$, rs2281827 and rs9508021 in VEGFR-1, and rs4145836 and rs9973653 in EPAS1; Supplementary Table $4 b$ ). Notably, bevacizumab treatment was again not identified as one of the 9 clinical covariates. When this OS signature was evaluated in the validation set, the HR for OS comparing above versus below the median was substantial and significant (HR 0.38; $95 \%$ CI $0.32-0.46$; $P<2 \times 10^{-16}$; Supplementary Fig. 2). The performance of the OS signature was also investigated with respect to PFS in the validation set and showed a similarly strong effect (HR 0.52; $95 \%$ CI 0.44-0.60; $P=3.3 \times 10^{-16}$ ).

Functional effects of variants predictive of bevacizumab outcome

To test whether any of these significant SNPs also affect mRNA expression of their respective genes, we assessed whether they were located in expression quantitative trait loci (eQTL), as identified by Genevar [34]. Using mRNA expression data from 856 lymphoblastoid cell lines [33], we confirmed that rs699946 in VEGF-A was located in an eQTL ( $P=0.0161$; Fig. 5a). In particular, rs699946 AA carriers exhibited a 6 and $12 \%$ reduction in mRNA expression compared to, respectively, rs699946 GA and GG carriers. In contrast, rs2234671 and rs12510099 were not located in an eQTL associated from IL8RA or VEGF$C$. Several of the EPAS1 SNPs did, however, locate in an eQTL from EPAS1. In particular, although the predictive rs4953344 variant in EPAS1 itself was not genotyped, the SNP with the highest $r^{2}$ value to rs4953344, i.e., rs3768727, was significantly associated with EPASI expression $(P=0.0437$; Fig. $5 b)$. Also, the minor rs13409493 T-allele, which was predictive in the metaanalysis and correlated with improved PFS (Table 2), was significantly associated with increased EPAS1 mRNA expression $(P=0.0326$; Fig. $5 b)$, whereas the rs 4145836 variant, which was associated with a prognostic effect, was not in an EPAS1 eQTL. On the other hand, we confirmed that rs1678607 and rs1642742 in VHL were both located in an eQTL significantly determining $V H L$ mRNA expression $\left(P=4.77 \times 10^{-8}\right.$ and $P=2.58 \times 10^{-4}$, respectively; Fig. 5c). In particular, minor alleles of both SNPs strongly correlated with increased VHL mRNA expression.

We observed a similar correlation between rs699946 and circulating plasma VEGF-A concentration in healthy 

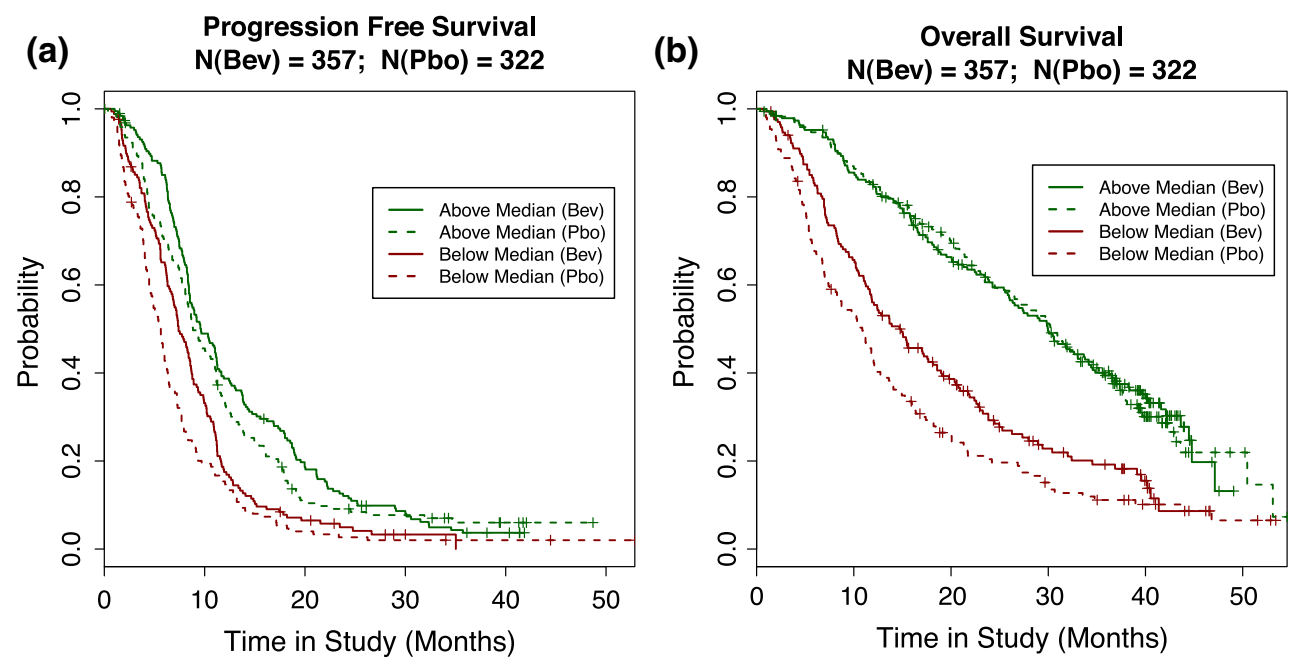

Fig. 4 Kaplan-Meier curves in the validation set classified based on the median of the signature for a PFS and $\mathbf{b}$ OS. The model was derived for PFS in a training set comprising half of the bevacizumab-treated and half of the placebo-treated patients

Fig. 5 Regional plots from the eQTL analysis in 856 lymphoblastoid cell lines of healthy female twins of the MuTHER study for a $V E G F-A$, b EPAS1, and c VHL. On the $X$ axis, the chromosomal positions of the SNPs are shown; on the $Y$-axis, the $-\log _{10} P$ value obtained for the eQTL analysis between the SNPs against mRNA expression of their respective genes is shown. Variants identified in this study, or their closest proxies genotyped in the MuTHER study, are indicated in the individual plots. d The correlation of VEGF plasma levels with SNP rs699946 genotypes. $B E V$ bevacizumab, $O S$ overall survival, $P F S$ progression-free survival, $P L A$ placebo
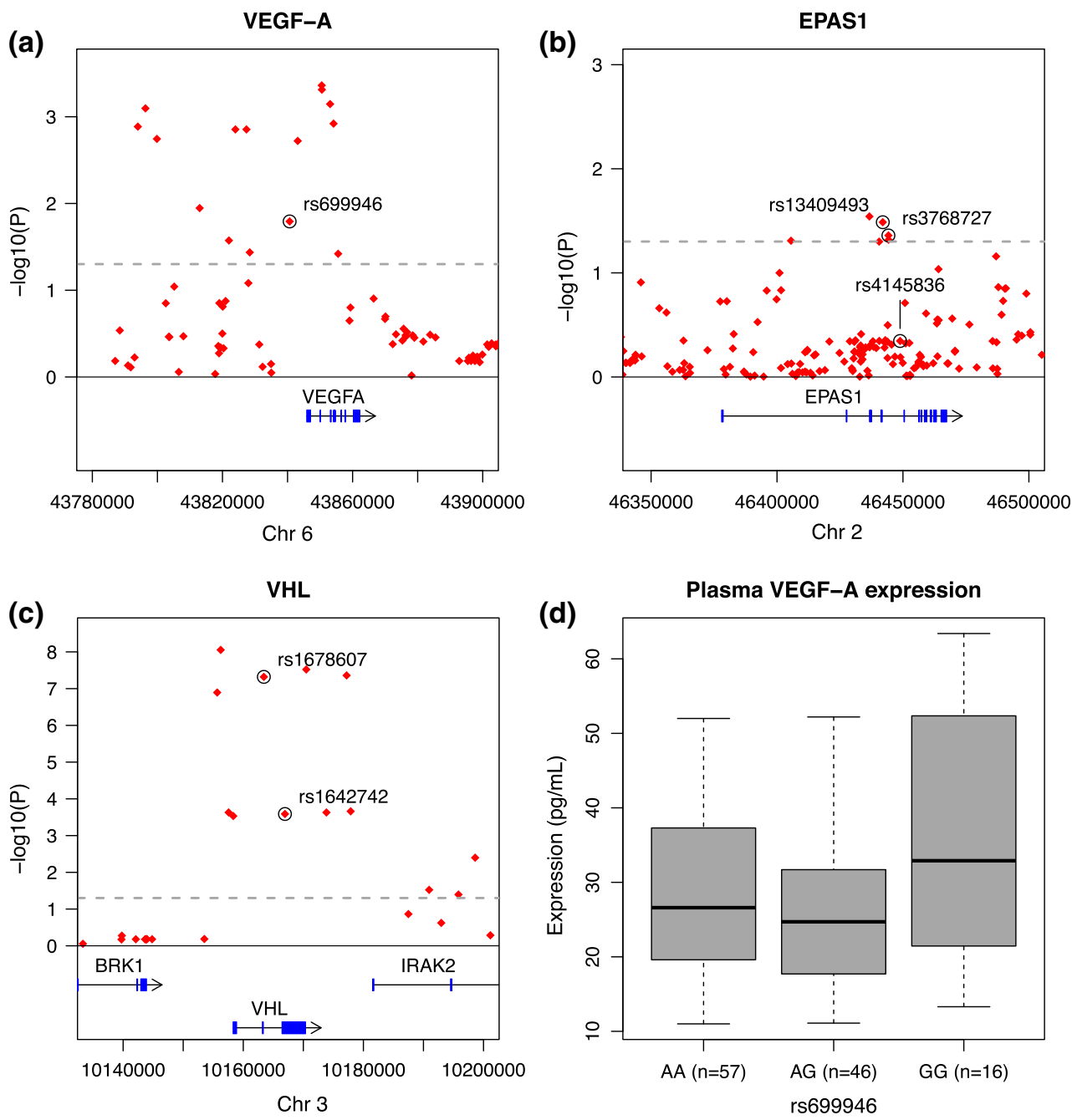
individuals $(P=0.006$; Fig. 5d) but not between rs12510099 and plasma VEGF-C concentration. EPAS1, VHL, and IL8RA were not detectable in plasma as these proteins are intracellular or membrane-bound.

\section{Discussion}

The most significant finding of this study is the identification of 3 genetic variants in VEGF-C, EPASI, and IL8RA that were predictive of bevacizumab treatment outcome. The effects of these variants were significant $(P<0.05)$ when assessing PFS in bevacizumab-treated patients only, as well as in a genotype-by-treatment interaction analysis. One additional variant in VEGF-A was also predictive in bevacizumab-treated patients but failed to reach significance in the interaction analysis $(P=0.091)$. This variant could nevertheless be considered as an additional variant potentially predictive of bevacizumab treatment outcome, as it significantly affected VEGF expression. Two variants, rs12505758 in VEGFR-2 and rs7987649 in VEGFR-1, were also predictive for OS. The interpretation of OS data was, however, more difficult as crossover events might have affected the OS analysis. It should be noted that none of the treatment-by-interaction effects for PFS or OS remained statistically significant after FDR correction for testing 195 variants. Replication in additional studies is therefore needed before considering these markers as true predictors of bevacizumab treatment outcome across tumor types.

A correlation between rs 2234671 in IL8RA (CXCRI) and treatment outcome has previously been described in a single-arm study of 132 patients receiving bevacizumab for colorectal cancer [22]. Wild-type CC carriers were characterized by increased response rates and prolonged PFS. In our much larger cohort of 716 bevacizumab-treated patients, similar effects on PFS were noticed, but importantly, rs2234671 was also significant in the treatment-byinteraction analysis including 686 patients receiving placebo. The rs2234671 variant represents a non-synonymous SNP in exon 1 of $C X C R 1$, which tags a large linkage disequilibrium block across $C X C R 1$. Although rs2234671 did not correlate with altered $C X C R 1$ mRNA expression in Genevar, it might still reduce IL8 signaling at the protein level by introducing an amino acid substitution (S276T) at a conserved position. Notably, altered expression of $C X C R 1$ has previously been reported to regulate angiogenesis independently of VEGF-A and to activate a tumorspecific immune response by attracting leukocytes, potentially suggesting mechanisms by which this variant might affect bevacizumab treatment outcome [41].

The rs699946 variant, which is located in the VEGF$A$ promoter, was previously identified as a marker of treatment outcome in patients receiving bevacizumab for neovascular age-related macular degeneration [42]. In the present study, AA carriers of rs699946 exhibited an improved PFS after bevacizumab treatment compared with placebo-treated patients. When stratifying VEGF plasma concentrations from healthy subjects according to rs699946, we noticed that VEGF concentrations were lower in AA than GG carriers, an effect that was confirmed at the mRNA level in Genevar. On the other hand, rs12510099 in $V E G F-C$ has not yet been studied and since it did also not affect $V E G F-C$ expression, its role in predicting bevacizumab treatment outcome is currently unclear. Finally, we also identified 2 variants in $V H L$ that were associated with bevacizumab treatment outcome at $P<0.05$. In the genewise analysis, the combined effects of both these variants were significant even at an FDRadjusted $q<0.05$ for PFS. Minor alleles of both variants also correlated with increased VHL mRNA expression in Genevar and were associated with improved PFS after bevacizumab.

Another intriguing finding is that, although several variants in EPAS1 were significant $(P<0.05)$ when assessing PFS in bevacizumab-treated patients only, one variant in EPAS1 exerted a strong prognostic rather than predictive effect on PFS. The prognostic effect of the rs4145836 variant resisted correction for multiple testing and was also retrieved in the multivariate signature identified by elastic net analyses. EPAS1 (or HIF-2 $\alpha$ ) is an oxygen-sensitive transcription factor that allows adaptation of cells to hypoxic environments [43]. It has a wellestablished role during angiogenesis [44-46], but recent evidence also implicates this gene as a key mediator of the metabolic adaption of tumors and the infiltration of inflammatory cells into the tumor microenvironment [47]. Previous studies revealed, for instance, that EPAS1, as assessed by immunohistochemistry, is present at increased levels in some tumors and that these patients have significantly decreased survival compared with patients whose tumor samples have undetectable levels of EPAS1 [48]. Furthermore, EPAS1 expression appears to be upregulated in the surrounding stroma, in particular in tumor-associated macrophages, as opposed to the tumor cells [49]. These elevated expression levels of EPAS1 in tumor-associated macrophages correspond directly with clinical severity of many different human cancers [50]. Interestingly, several genome-wide studies conducted in populations biologically adapted to living at high altitude, such as the Tibetan population, revealed EPAS1 variants that were under strong genetic selection and responsible for high-altitude adaptations [51-53]. Some of the variants identified in the present study were in high linkage disequilibrium with these variants supporting the notion that they affect EPASI expression or function. Genevar analysis indeed confirmed that several of these SNPs were highly significantly 
correlated with EPAS1 mRNA expression levels. Additional fine-mapping studies should be conducted to identify the causal variant(s) in EPASl contributing to these phenotypes. With respect to the latter, carriers of EPASI genotypes that correlated increased EPAS1 expression exhibited prolonged PFS.

Finally, using elastic net analyses to identify genetic variants and clinical variables significantly contributing to clinical outcome, we observed that bevacizumab treatment did not significantly contribute to the regression model, whereas, surprisingly, several of the genetic variants did contribute. The lack of an association with bevacizumab treatment can probably be explained by the fact that bevacizumab exerts very minimal therapeutic effects in some of the cancer types included in the meta-analysis, whereas, on the other hand, the fact that variants in angiogenesisrelated genes were identified as prognostic factors across various cancer types is noteworthy.

With respect to the statistical analysis, we chose to apply a pooled meta-analysis of individual patient data rather than a random-effects meta-analysis. Although the latter model is intuitively appealing, there were insufficient clinical studies to precisely model the between-studies variance. Another potential weakness of the study is the lack of a replication set. We decided, however, to combine all available data into a single analysis to maximize statistical power. Moreover, in the case of the association between PFS and rs4145836, the effect size estimated from pooling individual patient data was HR 0.74 , very close to that derived from the fixed-effects meta-analysis of summary statistics (HR 0.75), suggesting that the association was not attributable to patient stratification. Finally, since we selected 195 variants that were in low LD with each other, we did not perform a haplotype-based analysis, as has previously been performed, for instance, for the VEGF$A$ promoter [54, 55].

In conclusion, by performing a meta-analysis of individual data from more than 1,400 patients across 6 different tumor types, we identified several potentially predictive markers of bevacizumab treatment outcome that also functionally affected the expression levels of their target genes. We anticipate that these findings will contribute to ongoing efforts aimed at identifying markers that predict which patients will benefit most from bevacizumab therapy.

Acknowledgments We thank all patients who volunteered to participate in the genetic biomarker protocol of these studies and the research staff at the Vesalius Research Center, in particular Gilian Peuteman, Dominiek Smeets, and Thomas Van Brussel. The trials included in this analysis were sponsored and funded by F. HoffmannLa Roche, Basel, Switzerland. Funding for statistical analyses and third-party medical writing support for this paper were also provided by F. Hoffmann-La Roche. Sanne de Haas and Paul Delmar are employees of F. Hoffmann-La Roche Ltd. Matthieu Moisse is supported by the Fund for Scientific Research Flanders (FWO). The work of Peter Carmeliet is funded by long-term structural funding Methusalem by the Flemish Government. Diether Lambrechts is supported by the Seventh Framework Programme of the European Community for Research (AngioPredict). The trials included in this analysis were sponsored and funded by F. Hoffmann-La Roche, Basel, Switzerland. Funding for statistical analyses and third-party medical writing support for this paper were also provided by F. Hoffmann-La Roche.

Conflict of interest Sanne de Haas and Paul Delmar are employees of F. Hoffmann-La Roche, Basel, Switzerland. Aruna T. Bansal is a paid consultant of F. Hoffmann-La Roche. Eric Van Cutsem, Diether Lambrechts, and Peter Carmeliet have received research funding from F. Hoffmann-La Roche related to research into biomarkers for bevacizumab. David Miles has received honoraria from F. Hoffmann-La Roche for advisory boards and speaker engagements. Celine Pallaud is a former employee of F. Hoffmann-La Roche, Basel, Switzerland. Stefan Scherer is a former employee of Genentech. The remaining authors have declared no potential conflict of interest.

\section{References}

1. Hurwitz H, Fehrenbacher L, Novotny W et al (2004) Bevacizumab plus irinotecan, fluorouracil, and leucovorin for metastatic colorectal cancer. N Engl J Med 350(23):2335-2342. doi:10.1056/NEJMoa032691

2. Sandler A, Gray R, Perry MC et al (2006) Paclitaxel-carboplatin alone or with bevacizumab for non-small-cell lung cancer. N Engl J Med 355(24):2542-2550. doi:10.1056/NEJMoa061884

3. Miller K, Wang ML, Gralow J et al (2007) Paclitaxel plus bevacizumab versus paclitaxel alone for metastatic breast cancer. New Engl J Med 357(26):2666-2676. doi:10.1056/Nejmoa07 2113

4. Miles DW, Chan A, Dirix LY et al (2010) Phase III study of bevacizumab plus docetaxel compared with placebo plus docetaxel for the first-line treatment of human epidermal growth factor receptor 2-negative metastatic breast cancer. J Clin Oncol 28(20):3239-3247. doi:10.1200/JCO.2008.21.6457

5. Robert NJ, Dieras V, Glaspy J et al (2011) RIBBON-1: randomized, double-blind, placebo-controlled, phase III trial of chemotherapy with or without bevacizumab for first-line treatment of human epidermal growth factor receptor 2-negative, locally recurrent or metastatic breast cancer. J Clin Oncol 29(10):1252-1260. doi:10.1200/Jco.2010.28.0982

6. Escudier B, Pluzanska A, Koralewski P et al (2007) Bevacizumab plus interferon alfa-2a for treatment of metastatic renal cell carcinoma: a randomised, double-blind phase III trial. Lancet 370(9605):2103-2111. doi:10.1016/S0140-6736(07)61904-7

7. Burger RA, Brady MF, Bookman MA et al (2011) Incorporation of bevacizumab in the primary treatment of ovarian cancer. N Engl J Med 365(26):2473-2483. doi:10.1056/NEJMoa1104390

8. Perren TJ, Swart AM, Pfisterer J et al (2011) A phase 3 trial of bevacizumab in ovarian cancer. $N$ Engl J Med 365(26): 2484-2496. doi:10.1056/NEJMoa1103799

9. Aghajanian C, Blank SV, Goff BA et al (2012) OCEANS: a randomized, double-blind, placebo-controlled phase III trial of chemotherapy with or without bevacizumab in patients with platinum-sensitive recurrent epithelial ovarian, primary peritoneal, or fallopian tube cancer. J Clin Oncol 30(17):2039-2045. doi: $10.1200 / J c 0.2012 .42 .0505$ 
10. Henriksoon R, Bottomley A, Mason W, Saran F, Wick W, Nishikawa R (2013) Progression-free survival and health-related quality of life in AVAglio, a phase III study of bevacizumab, temozolomide, and radiotherapy in newly diagnosed glioblastoma. J Clin Oncol 31 (Suppl. abstr 2005). http://meetinglibrary. asco.org/content/114612-132

11. Maru D, Venook AP, Ellis LM (2013) Predictive biomarkers for bevacizumab: are we there yet? Clin Cancer Res 19(11): 2824-2827. doi:10.1158/1078-0432.Ccr-12-3409

12. Sennino B, McDonald DM (2012) Controlling escape from angiogenesis inhibitors. Nat Rev Cancer 12(10):699-709. doi:10. 1038/Nrc3366

13. Bergers G, Hanahan D (2008) Modes of resistance to antiangiogenic therapy. Nat Rev Cancer 8(8):592-603. doi:10.1038/ $\mathrm{Nrc} 2442$

14. Eichten A, Adler AP, Cooper B et al (2013) Rapid decrease in tumor perfusion following VEGF blockade predicts long-term tumor growth inhibition in preclinical tumor models. Angiogenesis 16(2):429-441. doi:10.1007/s10456-012-9328-3

15. Lambrechts D, Lenz HJ, de Haas S, Carmeliet P, Scherer SJ (2013) Markers of response for the antiangiogenic agent bevacizumab. J Clin Oncol 31(9):1219-1230. doi:10.1200/Jco.2012. 46.2762

16. Brauer MJ, Zhuang GL, Schmidt M et al (2013) Identification and analysis of in vivo VEGF downstream markers link VEGF pathway activity with efficacy of anti-VEGF therapies. Clin Cancer Res 19(13):3681-3692. doi:10.1158/1078-0432.Ccr-123635

17. Hegde PS, Jubb AM, Chen DF et al (2013) Predictive impact of circulating vascular endothelial growth factor in four phase III trials evaluating bevacizumab. Clin Cancer Res 19(4):929-937. doi:10.1158/1078-0432.Ccr-12-2535

18. Loupakis F, Cremolini C, Fioravanti A et al (2011) Pharmacodynamic and pharmacogenetic angiogenesis-related markers of first-line FOLFOXIRI plus bevacizumab schedule in metastatic colorectal cancer. Br J Cancer 104(8):1262-1269. doi:10.1038/ Bjc. 2011.85

19. Zhang W, Dahlberg SE, Yang D et al (2009) Genetic variants in angiogenesis pathway associated with clinical outcome in NSCLC patients (pts) treated with bevacizumab in combination with carboplatin and paclitaxel: subset pharmacogenetic analysis of ECOG 4599 [abstr 8032]. J Clin Oncol 27(15 Suppl.):414s. http://meetinglibrary.asco.org/content/33186-65

20. Schneider BP, Wang M, Radovich M et al (2008) Association of vascular endothelial growth factor and vascular endothelial growth factor receptor-2 genetic polymorphisms with outcome in a trial of paclitaxel compared with paclitaxel plus bevacizumab in advanced breast cancer: ECOG 2100. J Clin Oncol 26(28):4672-4678. doi:10.1200/JCO.2008.16.1612

21. Jubb AM, Harris AL (2010) Biomarkers to predict the clinical efficacy of bevacizumab in cancer. Lancet Oncol 11(12):1172-1183. doi:10.1016/S1470-2045(10)70232-1

22. Gerger A, El-Khoueiry A, Zhang W et al (2011) Pharmacogenetic angiogenesis profiling for first-line bevacizumab plus oxaliplatinbased chemotherapy in patients with metastatic colorectal cancer. Clin Cancer Res 17(17):5783-5792. doi:10.1158/1078-0432. CCR-11-1115

23. Lambrechts D, Claes B, Delmar P et al (2012) VEGF pathway genetic variants as biomarkers of treatment outcome with bevacizumab: an analysis of data from the AViTA and AVOREN randomised trials. Lancet Oncol 13(7):724-733. doi:10.1016/ S1470-2045(12)70231-0

24. Hansen TF, Christensen RD, Andersen RF, Spindler KLG, Johnsson A, Jakobsen A (2012) The predictive value of single nucleotide polymorphisms in the VEGF system to the efficacy of first-line treatment with bevacizumab plus chemotherapy in patients with metastatic colorectal cancer results from the Nordic ACT trial. Int J Colorectal Dis 27(6):715-720. doi:10.1007/ S00384-011-1382-6

25. Schneider BP, Gray RJ, Radovich M et al (2013) Prognostic and predictive value of tumor vascular endothelial growth factor gene amplification in metastatic breast cancer treated with paclitaxel with and without bevacizumab; results from ECOG 2100 trial. Clin Cancer Res 19(5):1281-1289. doi:10.1158/1078-0432.Ccr-12-3029

26. Loupakis F, Cremolini C, Dongyun Y et al (2013) Prospective validation of candidate SNPs of VEGF/VEGFR pathway in metastatic colorectal cancer patients treated with first-line FOLFIRI plus bevacizumab. Plos One 8(7):e66774. doi:10.1371/ journal.pone.0066774

27. Van Cutsem E, Vervenne WL, Bennouna J et al (2009) Phase III trial of bevacizumab in combination with gemcitabine and erlotinib in patients with metastatic pancreatic cancer. J Clin Oncol 27(13):2231-2237. doi:10.1200/JCO.2008.20.0238

28. Reck M, von Pawel J, Zatloukal P et al (2009) Phase III trial of cisplatin plus gemcitabine with either placebo or bevacizumab as first-line therapy for nonsquamous non-small-cell lung cancer: AVAiL. J Clin Oncol 27(8):1227-1234. doi:10.1200/Jco.2007. 14.5466

29. Ohtsu A, Shah MA, Van Cutsem E et al (2011) Bevacizumab in combination with chemotherapy as first-line therapy in advanced gastric cancer: a randomized, double-blind, placebo-controlled phase III study. J Clin Oncol 29(30):3968-3976. doi:10.1200/Jco. 2011.36.2236

30. Barret JC (2009) Haploview: visualization and analysis of SNP genotype data. Cold Spring Harb Protoc 10:pdb.ip71. doi:10. 1101/pdb.ip71

31. Pettersson FH, Anderson CA, Clarke GM et al (2009) Marker selection for genetic case-control association studies. Nat Protoc 4(5):743-752. doi:10.1038/Nprot.2009.38

32. Lambrechts D, Moisse M, Delmar P et al (2014) Genetic markers of bevacizumab-induced hypertension. Angiogenesis. doi:10. 1007/s10456-014-9424-7

33. Grundberg E, Small KS, Hedman AK et al (2012) Mapping cisand trans-regulatory effects across multiple tissues in twins. Nat Genet 44(10):1084-1089. doi:10.1038/ng.2394

34. Yang TP, Beazley C, Montgomery SB et al (2010) Genevar: a database and Java application for the analysis and visualization of SNP-gene associations in eQTL studies. Bioinformatics 26(19):2474-2476. doi:10.1093/bioinformatics/btq452

35. Benjamini Y, Hochberg Y (1995) Controlling the false discovery rate-a practical and powerful approach to multiple testing. J R Stat Soc B 57(1):289-300

36. Goeman JJ, van de Geer SA, de Kort F, van Houwelingen HC (2004) A global test for groups of genes: testing association with clinical outcome. Bioinformatics 20:93-99

37. Goeman JJ, Oosting J, Cleton-Jansen AM, Anninga JK, van Houwelingen HC (2005) Testing association of a pathway with survival using gene expression data. Bioinformatics 21(9):1950-1957. doi:10.1093/Bioinformatics/Bti267

38. Zou H, Hastie T (2005) Regularization and variable selection via the elastic net. J R Stat Soc B 67:301-320. doi:10.1111/J.14679868.2005.00503.X

39. Friedman J, Hastie T, Tibshirani R (2008) Regularization paths for generalized linear models via coordinate descent. J Stat Soft $33: 1-22$

40. Simon N, Friedman J, Hastie T, Tibshirani R (2011) Regularization paths for Cox's proportional hazards model via coordinate descent. J Stat Soft 39:1-13

41. Choi I, Lee YS, Chung HK et al (2013) Interleukin-8 reduces post-surgical lymphedema formation by promoting lymphatic vessel regeneration. Angiogenesis 16(1):29-44. doi:10.1007/ s10456-012-9297-6 
42. Nakata I, Yamashiro K, Nakanishi H, Tsujikawa A, Otani A, Yoshimura N (2011) VEGF gene polymorphism and response to intravitreal bevacizumab and triple therapy in age-related macular degeneration. Jpn J Ophthalmol 55(5):435-443. doi:10.1007/ S10384-011-0061-Z

43. Chen J, Michan S, Juan AM et al (2013) Neuronal sirtuin1 mediates retinal vascular regeneration in oxygen-induced ischemic retinopathy. Angiogenesis 16(4):985-992. doi:10.1007/ s10456-013-9374-5

44. Xia GB, Kageyama Y, Hayashi T, Kawakami S, Yoshida M, Kihara M (2001) Regulation of vascular endothelial growth factor transcription by endothelial PAS domain protein 1 (EPAS1) and possible involvement of EPAS1 in the angiogenesis of renal cell carcinoma. Cancer 91(8):1429-1436. doi:10.1002/10970142(20010415)91:8<1429:Aid-Cncr1149>3.0.Co;2-V

45. Favier J, Plouin PF, Corvol P, Gasc JM (2002) Angiogenesis and vascular architecture in pheochromocytomas-distinctive traits in malignant tumors. Am J Pathol 161(4):1235-1246. doi:10.1016/ S0002-9440(10)64400-8

46. Favier J, Lapointe S, Maliba R, Sirois MG (2007) HIF2 alpha reduces growth rate but promotes angiogenesis in a mouse model of neuroblastoma. BMC Cancer 7. doi:10.1186/1471-2407-7-139

47. Keith B, Johnson RS, Simon MC (2011) HIF1 $\alpha$ and HIF2 $\alpha$ : sibling rivalry in hypoxic tumour growth and progression. Nat Rev Cancer 12:9-22

48. Jubb AM, Turley H, Moeller HC et al (2009) Expression of deltalike ligand 4 (Dl14) and markers of hypoxia in colon cancer. Br J Cancer 101(10):1749-1757. doi:10.1038/Sj.Bjc.6605368
49. Talks KL, Turley H, Gatter KC et al (2000) The expression and distribution of the hypoxia-inducible factors HIF-1 alpha and HIF-2 alpha in normal human tissues, cancers, and tumor-associated macrophages. Am J Pathol 157(2):411-421. doi:10.1016/ S0002-9440(10)64554-3

50. Shay JES, Simon MC (2012) Hypoxia-inducible factors: crosstalk between inflammation and metabolism. Semin Cell Dev Biol 23(4):389-394. doi:10.1016/J.Semcdb.04.004

51. Simonson TS, Yang YZ, Huff CD et al (2010) Genetic evidence for high-altitude adaptation in Tibet. Science 329(5987):72-75. doi:10.1126/Science.1189406

52. Yi X, Liang Y, Huerta-Sanchez E et al (2010) Sequencing of 50 human exomes reveals adaptation to high altitude. Science 329(5987):75-78. doi:10.1126/Science.1190371

53. Beall CM, Cavalleri GL, Deng LB et al (2010) Natural selection on EPAS1 (HIF2 alpha) associated with low hemoglobin concentration in Tibetan highlanders. Proc Natl Acad Sci USA 107(25):11459-11464. doi:10.1073/Pnas.1002443107

54. Lambrechts D, Storkebaum E, Morimoto M et al (2003) VEGF is a modifier of amyotrophic lateral sclerosis in mice and humans and protects motoneurons against ischemic death. Nat Genet 34(4):383-394. doi:10.1038/ng1211

55. Han SW, Kim GW, Seo JS et al (2004) VEGF gene polymorphisms and susceptibility to rheumatoid arthritis. Rheumatology (Oxf) 43(9):1173-1177. doi:10.1093/rheumatology/keh281 